\title{
Vocación de carrera en académicos de una universidad pública en Argentina
}

Lucas Pujol-Cols y Mariana Lazzaro-Salazar

\section{RESUMEN}

Este estudio indaga las percepciones que un grupo de académicos argentinos tiene sobre el rol de la vocación en su carrera, con el objetivo de ofrecer reflexiones que ayuden a enriquecer nuestra comprensión de las dimensiones que componen este complejo constructo en el contexto académico. Se realizaron entrevistas semiestructuradas a 22 académicos de una universidad pública argentina y se efectuó un análisis del contenido de sus discursos desde una perspectiva crítica. Los resultados de esta investigación permitieron identificar una serie de categorías que ayudan a operacionalizar empíricamente el concepto de vocación para la carrera académica.

Palabras clave: vocación, discursos, docencia, profesores, Argentina.

\section{Lucas Pujol-Cols}

Argentino. Doctor en Administración, Universidad Nacional del Centro de la Provincia de Buenos Aires (UNICEN), Argentina. Investigador de Postdoctorado, Consejo Nacional de Investigaciones Científicas y Técnicas, Argentina y Universidad Católica del Maule, Chile. Temas de investigación: diseño del trabajo, riesgos psicosociales, trabajo emocional, recursos personales, bienestar en el trabajo, salud ocupacional. ORCID: 0000-0002-8754-3416.

\section{Mariana Lazzaro-Salazar}

lazzaromari@gmail.com Argentina. Doctora en Lingüística, Victoria University of Wellington, Nueva Zelanda. Académica Auxiliar, Centro de Investigación de Estudios Avanzados, Vicerrectoría de Investigación y Postgrado, Universidad Católica del Maule, Chile. Temas de investigación: discurso y sociedad, análisis del discurso, sociolingüística, discurso y salud. ORCID: 0000-0001-7624-0068. 
Vocação de carreira em acadêmicos de uma universidade pública na Argentina

\title{
RESUMO
}

Este estudo indaga as percepções que um grupo de acadêmicos argentinos tem sobre a função da vocação em sua carreira, com o objetivo de oferecer reflexões que ajudem a enriquecer nossa compreensão das dimensões que compõem este complexo constructo no contexto acadêmico. Se realizaram entrevistas semiestruturadas a 22 acadêmicos de uma universidade pública argentina e se efetuou uma análise do conteúdo de seus discursos partindo de uma perspectiva crítica. Os resultados desta pesquisa permitiram identificar uma serie de categorias que ajudam a operacionalizar empiricamente o conceito de vocação para a carreira acadêmica.

Palavras chave: Palavras chave: vocação, discursos, docência, professores, Argentina.

\section{Career vocation of the academic staff at a public university in Argentina}

\begin{abstract}
This study analyzes the perceptions that a group of Argentine university staff have about the role of vocation in their careers, with the aim of offering reflections that help to enrich our understanding of the dimensions that make up this complex construct in the academic context. Semi-structured interviews were conducted with 22 academics from an Argentine public university and a content analysis of their discourses was carried out from a critical perspective. The results of this research allowed us to identify a series of categories that help to operationalize empirically the concept of vocation for an academic career.
\end{abstract}

Keywords: vocation, discourses, teaching, professors, Argentina. 


\section{Introducción}

Numerosas investigaciones coinciden en que la docencia universitaria constituye una de las profesiones más estresantes a nivel mundial (Extremera et al., 2010a). Desde el punto de vista del contenido del rol, los académicos enfrentan elevadas demandas psicológicas (Salanova et al., 2005), en virtud de la complejidad y variedad de actividades que suelen desempeñar, en general, en el marco de fuertes restricciones temporales y asincronías en el flujo de trabajo (por ejemplo, planificar clases, liderar o participar en proyectos de investigación y/o transferencia tecnológica, supervisar y formar recursos humanos, entre muchas otras). Estas actividades, además, requieren altos niveles de concentración, precisión y atención a los detalles, consumiendo, como resultado, elevada energía cognitiva (Extremera et al., 2010b). Al mismo tiempo, la profesión académica involucra también importantes demandas emocionales, es decir, aspectos del trabajo que requieren un esfuerzo emocional sostenido (Yin et al., 2013), las cuales, en caso de conducir a un estado de disonancia emocional, pueden resultar en fuertes presiones psicosociales (Goh et al., 2015).

De particular interés para este estudio, la profesión académica en Argentina no es ajena a las problemáticas identificadas por esta literatura internacional. No obstante, a las anteriores se adicionan otras de índole local, tales como los incrementos sostenidos en las tasas de matriculación estudiantil en tiempos de restricciones presupuestarias, el acceso limitado a recursos físicos, la falta de una estructura salarial que compense adecuadamente los esfuerzos, el escaso apoyo social y las débiles oportunidades de desarrollo de carrera (Collado et al., 2016; Pujol-Cols y Lazzaro-Salazar, 2018). Este contexto ocupacional caracterizado por elevadas demandas laborales e insuficientes recursos organizacionales, ciertamente eleva los riesgos psicosociales a los que se encuentran expuestos los académicos argentinos. Estos últimos representan aquellos factores relacionados con el trabajo que, sea por exceso, defecto o combinación, constituyen una amenaza para la integridad física, social y/o psicológica de los individuos (Meliá et al., 2006).

Sorprendentemente, a pesar de los elevados riesgos psicosociales que caracterizan la profesión académica, numerosos estudios a nivel internacional han demostrado que la mayoría de los académicos no reportan sentirse agotados, desmotivados o insatisfechos en su trabajo, sino que, por el contrario, tienden a experimentar estados muy positivos (Shin y Jung, 2014). Esta situación, incluso, ha sido observada en el contexto argentino. Por ejemplo, algunos estudios previos han demostrado que, si bien los académicos parecieran reconocer la existencia de una alta prevalencia de demandas psicológicas, conflicto trabajo-familia e insuficiente estima en su trabajo, al mismo tiempo, tienden a experimentar un elevado work engagement y satisfacción laboral (Pujol-Cols y Arraigada, 2017; Pujol-Cols y Lazzaro-Salazar, 2018). Estos hallazgos resultan similares a los reportados en un estudio intercultural publicado en Times Higher Education (2010), el cual revela que Argentina es, junto con México y Malasia, uno de los países donde los académicos verifican los niveles promedio más altos de satisfacción laboral.

Esta aparente inconsistencia entre la naturaleza estresante del trabajo y las actitudes y percepciones de los académicos conduce a preguntarse qué otras terceras variables y/o procesos podrían estar influyendo en esta relación de modo que los académicos, en promedio, tiendan a experimentar estados positivos, incluso frente a la presencia de condiciones de trabajo altamente estresantes. En un intento por dar respuesta a este interrogante, la teoría de demandas y recursos laborales (en adelante, D-RL) propone que los recursos personales, entendidos como aquellas autoevaluaciones positivas vinculadas a la resiliencia que se refieren a la percepción de los individuos acerca de su capacidad para controlar e influir en el entorno (Avey et al., 2011), asumen un rol fundamental en la manera en la que los individuos hacen frente a los riesgos psicosociales a los que se 
encuentran expuestos en su trabajo (Xanthopoulou et al., 2009). La personalidad ciertamente representa uno de los recursos personales más ampliamente estudiados en esta línea de investigación. Sin embargo, el constructo que ha concentrado mayor atención en el marco de la teoría D-RL recibe el nombre de capital psicológico positivo (Luthans y Youssef, 2004), un concepto de orden superior que comprende la esperanza, la resiliencia, el optimismo y la autoeficacia (ver Newman et al., 2014 para una revisión reciente). Así, numerosos estudios han demostrado de modo consistente que los recursos personales tienen la capacidad de mitigar los efectos negativos de varios factores de riesgo psicosocial sobre la salud ocupacional (fenómeno que ha sido denominado "hipótesis de la reacción diferencial", ver Kammeyer-Mueller et al., 2009), al mismo tiempo que existe evidencia de que los recursos personales también son capaces de amplificar los efectos positivos de ciertos recursos organizacionales (Hentrich et al., 2017).

Otros estudios más recientes han propuesto una forma diferente, aunque complementaria, de abordar los efectos de los recursos personales, transladando el foco de atención desde las características de los individuos (como la personalidad o el capital psicológico positivo) hacia las características de la relación que éstos tienen con su trabajo. Es así que autores como Wrzesniewski et al. (1997) advierten que las personas pueden tener diferentes orientaciones hacia sus trabajos, señalando que existen carreras que no sólo representan una fuente de ingresos económicos o satisfacción, sino que también proporcionan un sentido de propósito que motiva su labor (Weir, 2013). Para explicar este fenómeno, algunos investigadores han recurrido al concepto de vocación de carrera (o career calling en inglés; Dobrow y Tosti-Kharas, 2012), el cual puede ser entendido como un llamado trascendental a asumir un cierto rol laboral, cuya motivación es primariamente prosocial y conduce a un profundo sentido de propósito y significado (Duffy y Dik, 2013). En efecto, las personas con altos grados de vocación de carrera no trabajan solamente para obtener una contraprestación económica o para avanzar en su carrera, sino más bien por la sensación de propósito que les brinda asumir un determinado rol laboral (Duffy et al., 2015).

La vocación de carrera ha sido examinada de múltiples maneras y desde diversos abordajes. $\mathrm{Al}$ respecto, algunos autores han distinguido conceptualmente entre la presencia (o sentimiento) de una determinada vocación de carrera y su búsqueda a través del tiempo (Duffy et al., 2012). Teniendo en cuenta estas distinciones conceptuales, Dik et al. (2012) desarrollaron el Calling and Vocation Questionnaire (CVQ), un instrumento de auto-reporte compuesto por 24 reactivos agrupados en seis dimensiones teóricas (es decir, búsqueda de un llamado trascendental, presencia de un llamado trascendental, búsqueda de un trabajo con propósito, presencia de un trabajo con propósito, búsqueda de una orientación prosocial y presencia de una orientación prosocial) que componen la vocación de carrera. Cabe señalar que el CVQ constituye uno de los instrumentos más empleados en la literatura sobre vocación de carrera por reflejar de forma satisfactoria los factores personales que la componen.

En este contexto, se ha demostrado que tener una vocación de carrera podría conducir a numerosos beneficios. Para empezar, existe evidencia que señala que la vocación tiende a acrecentar la sensación de significado, definido como el grado en que los individuos sienten que el trabajo les resulta personalmente significativo (Douglass et al., 2016). Asimismo, los individuos que experimentan esta sensación de propósito suelen estar más comprometidos con sus carreras, lo que no sólo tiende a estar asociado con mayores niveles de satisfacción (Littman-Ovadia y Steger, 2010) e identificación organizacional (Cardador et al., 2011), sino también con menores intenciones de rotación (Duffy et al., 2011). A pesar de que hasta el momento algunas investigaciones han logrado vincular con éxito la vocación de carrera con varios estados individuales y resultados organizacionales positivos, los 
factores que subyacen a la vinculación entre la vocación de carrera y el bienestar en el trabajo han sido limitadamente explorados en la literatura, particularmente en profesiones con altos niveles de presencia de riesgos psicosociales, como es el caso de los académicos (Duffy et al., 2011).

En este sentido, esta investigación pretende colaborar en dar respuesta a los numerosos estudios recientes que han advertido la necesidad de construir teoría en torno al concepto de vocación y, en particular, de examinar los factores por los que la vocación se encuentra asociada a estados positivos relacionados con la salud (Duffy et al., 2018). Con este propósito en mente, el presente estudio indaga las percepciones que académicos de una universidad pública en Argentina tienen con respecto al rol de la vocación en su labor profesional, con el objetivo de ofrecer reflexiones que ayuden a enriquecer nuestra comprensión y conocimiento de las dimensiones que componen la vocación de carrera. Mediante el análisis de los relatos de los participantes proponemos una serie de subdimensiones que ayudan a operacionalizar el concepto de vocación en la carrera académica, de modo de reflejar la complejidad del constructo en este contexto profesional.

\section{Métodos}

\section{Contexto de estudio y participantes}

El sistema argentino de educación superior ha sido definido como un "sistema orientado a la enseñanza”, puesto que la mayoría de los recursos financieros, físicos y humanos disponibles están destinados a apoyar actividades relacionadas con la enseñanza, en detrimento de otras actividades académicas como la investigación y la transferencia tecnológica (Shin y Jung, 2014). Como resultado, la mayoría de los académicos que trabajan en universidades argentinas tienen contratos de tiempo parcial y sólo un porcentaje significativamente menor posee un contrato de tiempo completo. ${ }^{1}$ En 2013, los contratos de tiempo parcial representaban más del $87 \%$ del total de contratos de las universidades nacionales (Secretaría de Políticas Universitarias, García de Fanelli, 2014). Sin embargo, es importante señalar que una cantidad significativa de académicos de tiempo parcial suele tener más de un contrato, en virtud de su desempeño como docentes en más de un curso (ver Pujol-Cols et al., 2019). También debe señalarse que los salarios de los docentes universitarios en Argentina se encuentran entre los más bajos del mundo (Rumbley et al., 2008) y que cerca del 30\% del personal académico que trabaja en las universidades públicas argentinas no recibe ninguna compensación monetaria por sus servicios, desempeñando cargos ad honorem (Marquina, 2012).

En este contexto, un total de 22 académicos de una universidad pública de la provincia de Buenos Aires participaron de manera voluntaria en este estudio. Los datos fueron recolectados siguiendo los principios de muestreo gradual y saturación teórica. El primero implicó que los participantes fueron seleccionados de modo progresivo conforme se avanzó en el proceso de recolección y análisis de datos. El segundo se logró en el momento en que el beneficio marginal (en términos de la emergencia de nuevas categorías relevantes para el problema de investigación) no compensó el costo de entrevistar a un informante adicional. Se utilizó una técnica de muestreo de bola de nieve, la cual ha sido ampliamente utilizada en estudios cualitativos previos sobre carreras profesionales (por ejemplo, Lazzaro-Salazar, 2019). El propósito de esta estrategia consistió en lograr una heterogeneidad de perfiles suficiente para brindar representatividad teórica a la muestra. De este modo, se procuró la participación de docentes con distinto género, edad, jerarquía, dedicación horaria (es decir, con contrato a tiempo parcial o completo) y área de desempeño.

${ }^{1}$ Teniendo en cuenta la elevada heterogeneidad que caracteriza la profesión académica en Argentina (para una descripción más detallada, ver Pujol-Cols et al., 2019), en este artículo se optó por utilizar los términos "académico" y "docente" indistintamente. 
Así, la muestra se compuso por 15 participantes mujeres y siete participantes hombres que se desempeñaban como docentes y/o investigadores en diferentes áreas del conocimiento (por ejemplo, Administración General, Administración Estratégica, Comportamiento Organizacional, Contabilidad, Economía, Turismo, Psicología Laboral, Psicología Clínica, Psicología Forense, entre otras). Con respecto a la edad, cuatro participantes tenían 30 años o menos, 10 entre 30 y 50 años, y ocho más de 50 años. En cuanto a la jerarquía del cargo de los participantes, 13 eran Profesores y nueve eran Auxiliares. Finalmente, sies participantes tenían dedicaciones exclusivas, tres dedicaciones semi-exclusivas y 13 dedicaciones simples.

\section{Recolección de datos}

Esta investigación utilizó un diseño cualitativo y la recolección de datos se realizó mediante entrevistas semiestructuradas, siguiendo las recomendaciones de Eisenhardt (1989) para la confección de un guión flexible. El mismo incluyó una serie de preguntas que se diseñaron en consideración de las dimensiones incluidas en el GVQ(Dik et al., 2012): búsqueda de un llamado trascendental, presencia de un llamado trascendental, búsqueda de un trabajo con propósito, presencia de un trabajo con propósito, búsqueda de una orientación prosocial y presencia de una orientación prosocial. Las preguntas fueron lo suficientemente generales como para posibilitar la emergencia espontánea de categorías de análisis. Así, el guión se compuso por tres partes. En la primera, se solicitó a los participantes que describan sus condiciones de trabajo y las tareas que realizan actualmente en la universidad. En la segunda, se los invitó a reflexionar sobre sus sentimientos y experiencias al desempeñar sus diferentes roles laborales. En la tercera, se les pidió que explorasen las razones que motivan su participación en la universidad. Las entrevistas duraron aproximadamente una hora cada una. Todas ellas fueron grabadas y, luego, transcritas textualmente (ver delineamientos en Lazzaro-Salazar, 2013).

\section{Análisis de los datos}

Se realizó un análisis de contenido de las entrevistas en consideración de los principios del análisis del discurso desde una perspectiva crítica, la cual nos permite analizar cómo los participantes construyen la realidad a través del lenguaje y considerar sus posicionamientos identitarios y las ideologías subyacentes en las opiniones de nuestros participantes (Mendoza y Lazzaro-Salazar, 2018). De esta forma, se identificaron temas emergentes y recurrentes de interés analítico y se seleccionaron ejemplos por su capacidad para ilustrar los temas centrales en relación con la vocación de carrera de estos académicos argentinos. Estos ejemplos se agruparon en las tres macro-categorías que según Dik et al. (2012) definen la vocación de carrera y la diferencian de otros conceptos con los que podría solaparse (como el gusto por el trabajo, por ejemplo). Así, las tres macro-categorías que se presentan son: 1) el reconocimiento de la vocación, 2) la orientación prosocial de la vocación, y 3) el sentido de la vocación. Nuestro análisis contribuye en identificar sub-categorías que ayuden a operacionalizar esas tres macro-categorías.

\section{Resultados}

\section{Reconocimiento de la vocación}

De acuerdo con los relatos de nuestros académicos, el reconocimiento de la vocación de carrera está dado por factores tanto internos como externos a la persona, que resultan en procesos espontáneos y/o intencionados de reconocimiento de la vocación (ver Duffy y Dik, 2009). Como resultado de ambos procesos de reconocimiento, los académicos desarrollan lazos de vinculación emocional con su profesión (aspecto emocional de la vocación), a la vez que descubren sus propias capacidades y competencias para el ejercicio profesional (aspecto cognitivo de la vocación). A continuación, se presentan las dimensiones del reconocimiento de la vocación. 


\section{Origen del reconocimiento}

Dentro del reconocimiento de la vocación se distinguen dos formas en las que los académicos reportan haber identificado su vocación y que, por consiguiente, dan origen a ésta. En primer lugar, lo que aquí denominamos el llamado vocacional parece estar dado por procesos involuntarios, no intencionados y espontáneos por los que la persona identifica su vocación. En segundo lugar, otro grupo de académicos explica atravesar una fase de descubrimiento de la vocación, es decir, una etapa de exploración y búsqueda intencionada en la que (comúnmente durante el curso de la formación universitaria) la persona busca y descubre su vocación al entrar en contacto formal con la carrera docente.

\section{Llamado vocacional}

Algunos participantes comentan que se dan cuenta de cuál es su vocación previamente a tener contacto formal con la carrera, surgiendo la misma como una motivación intrínseca que se manifiesta desde la niñez a través de la satisfacción personal que les genera la práctica lúdica de la profesión.

Yo le enseñaba a mi hermana a leer y escribir, porque tenía dos años menos que yo, a mis primos los sentaba y ellos aprendían conmigo todo, como siempre fui rápida para las matemáticas y demás le di clases particulares ad honorem a Dios y a María santísima. Entonces toda la vida [...] En el secundario los profesores decían "en vez de ir a una maestra particular que te enseñe [nombre de participante]". Entonces [...] Esto nace con uno, ¿entendés? (ejemplo 1 , entrevista 5$){ }^{2}$

En esta misma línea, la entrevista 11 menciona que esta vocación está inscrita en los genes, aludiendo a que una persona nace con este sentido de la vocación. Sin embargo, para otros la vocación se descubre recién al tener contacto con la educación superior, como se verá a continuación.

\section{Descubrimiento de la vocación}

Para estos participantes la vocación por la carrera docente fue identificada cuando comenzaron sus estudios universitarios y, en muchos de estos casos, cuando se comenzaron a involucrar en la vida académica de forma activa participando, por ejemplo, en ayudantías. Así, para este grupo de participantes el descubrimiento de la vocación nace a partir de la práctica de ésta en el ámbito académico.

\begin{abstract}
No, de chico no sabía que quería ser docente, pero sí, cuando entré a la Universidad y me di cuenta lo que era ser docente y demás (ejemplo 2, entrevista 9).

Desde cuando era alumna [en la universidad] participaba en espacios de docencia, o sea que estuvo ahí [...] estuvo como solapado y cuando me gradué continúe con la docencia (ejemplo 3, entrevista 10).
\end{abstract}

\section{Naturaleza del reconocimiento}

Como se puede apreciar en los ejemplos de arriba, para algunos académicos la naturaleza del reconocimiento de la vocación está dada por el descubrimiento de factores emocionales ligados a la vinculación afectiva que poseen con el rol docente, que se manifiestan en la elevada satisfacción que les genera el ejercicio de este último. Para otros, el reconocimiento está ligado al descubrimiento de factores cognitivos, como competencias, capacidades y habilidades, que determinan su potencial para el ejercicio efectivo de la carrera docente.

\section{Aspectos emocionales (sentimiento de vocación)}

Así, algunos participantes señalan que este reconocimiento trascendental de la vocación de carrera está

\footnotetext{
${ }^{2}$ Nota del editor. En las transcripciones se utilizarán corchetes para representar pausas o texto omitido, y letra cursiva para denotar énfasis en las expresiones.
} 
dado por aspectos emocionales ligados a su satisfacción personal, algo que viene con ellos de forma innata.

Cuando era chica [...] a mí no sé por qué me encantaba $[\ldots]$ yo ponía a todos mis muñecos en fila y les daba clase, no sé, me salía [...] me gustaba tomarles lista, a mis muñecos, les daba clase, de hecho cuando me compraron un pizarrón me gustaba escribir en el pizarrón [...] y yo tenía cinco años. O sea, eso desde el punto de vista, por ahí, emocional (ejemplo 4 , entrevista 18).

\section{Aspectos cognitivos (capacidades vocacionales)}

Mientras que algunos académicos reportan tener sentimientos vinculados al reconocimiento de la vocación, otros explican que esta última fue adquirida de forma más o menos paulatina a partir de diversas experiencias socio-culturales:

Desde chiquita, tal vez vienen estas cosas de la herencia familiar ¿no? Tengo, bueno [...] obviamente no las tengo más, primas hermanas de mi papá, maestras, directoras de escuela, y jugar con ellas, ver su escritorio, verlas trabajar, que me prestaran sus libros, era todo como el mundo ideal para mí [...] fue elegido, fue opción mía (ejemplo 5, entrevista 21).

A través de éstas, descubren tener habilidades y capacidades para el ejercicio de la profesión, lo que evidencia un aspecto cognitivo vinculado al reconocimiento de la vocación que también se comienza a manifestar desde la niñez y adolescencia:

Me acuerdo que una profesora de química [...] me dijo [...] en un oral $[\ldots]$ "vos tenés mucha pasta para la docencia" $[\ldots]$ por como hablaba. Bueno eso fue como que me terminó de convencer. Así que [...] siempre lo tuve de manera innata, siempre ayudaba a mis compañeros, siempre explicaba [...] Siempre mis compañeros me decían "vos sí tenés pasta para ser docente [...] es lo tuyo" (ejemplo 6, entrevista 5).

Yo por ejemplo preparaba a mis compañeros de secundario para que rindieran los finales de diciembre, marzo, venían todos a mi casa, y yo les explicaba, y ahí me di cuenta que no sólo me gustaba, sino que servía $[. .$.$] no sé si lo hacía bien o mal$ pero lo que hacía le servía a alguien, entonces ahí me di cuenta que yo por ahí podía tener potencial (ejemplo 7, entrevista 18).

Esta observación sobre 'tener pasta' o 'servir' para la profesión alude a la percepción de que estas capacidades (al igual que el sentimiento de vocación) son innatas para muchos de estos académicos.

\section{Orientación prosocial de la vocación}

Otro de los aspectos que componen la vocación es la orientación prosocial, la cual consiste en un acto voluntario de intentar ayudar al otro (Mesurado et al., 2019). Guando describimos la orientación prosocial de la vocación se debe tener en cuenta cómo esta acción beneficia a terceros, por un lado, y cómo apoya el desarrollo individual de la persona que realiza la acción, por el otro.

\section{Aporte ético-valórico}

En el caso de los académicos entrevistados para este estudio, el primer aspecto recién mencionado está dado por el aporte ético-valórico que la labor académica realiza a través del proceso de enseñanza y que se evidencia en un comportamiento (por parte de los estudiantes y graduados) acorde a estos valores. Así, los siguientes relatos reflexionan sobre cómo la enseñanza les da a estos académicos la oportunidad de formar profesionales en valores, pensamientos y acciones éticas con las que se sienten personalmente identificados (por ejemplo, pensamiento crítico-reflexivo, ética, libre albedrío) y las cuales son socialmente deseables y necesarias.

[ 
Mi función es] formar futuros profesionales desde lo ético, desde una actitud crítica, desde la función de pensarse ellos mismos como instrumentos principales, instrumentos de trabajo el día de mañana cuando se reciban $[\ldots]$ con el soporte técnico y teórico que brindan las herramientas y desde el trabajo de repensarse a sí mismos desde lo personal e inmersos en una institución [...] y en una sociedad determinada y en una época determinada (ejemplo 8, entrevista 10).

Algunos colaboran en programas de alfabetización en comedores, ayudan en la elaboración de las comidas y esa situación del rol es mucho más enriquecedora por ahí que algunos de los aprendizajes que nosotros podríamos ampliar. Podría ser como muy enriquecedor [...] Yo creo que es ese sentido esperanzador de cambio. Por mi propia historia personal, la educación ha sido y me ha dado otro lugar en el mundo (ejemplo 9, entrevista 14).

\section{Reflejo del altruismo}

En segundo lugar, la orientación prosocial también apoya el desarrollo personal de los académicos, quienes explican que realizan su labor académica por vocación y no por una motivación extrínseca ligada a la recepción de recompensas económicas y/o reconocimiento social.

Yo siempre trato de poner lo mejor de mí porque siento vocación por lo que hago. Cuando uno siente vocación da lo mejor de sí mismo, es ese sentir, ese llamado que va más allá de una simple profesión por una paga, por una remuneración, excede. Por eso te digo, no sé ni las horas que trabajo porque lo hago por vocación, realmente (ejemplo 10, entrevista 19).

Me parece que es lo que se necesita para poder ser docente, la docencia no tiene $[. .$.$] un recono-$ cimiento ni social ni económico, muchas veces ni siquiera en cuanto al estatus académico, lo que se necesita es estar convencido de que es necesario y es lo que a uno le gusta (ejemplo 11, entrevista 11).
Imagínate que acá están trabajando prácticamente ad honorem. Hay 7 personas en un equipo que trabaja ad honorem [...] o que están con becas de formación [...] y nadie les tiene que pedir que cumplan horario [...] están a las 10 de la noche atendiendo un caso. Qué puedo decirte del equipo, es un lujo, un honor que me acompañen en las movidas que hacemos, que no lo hacen por mí, lo hacen porque están convencidos de que favorecer las condiciones de posibilidad para procesos subjetivantes, es algo que es parte de nuestra función (ejemplo 12, entrevista 17).

En estos relatos se puede destacar la importancia de la entrega personal y profesional en la labor académica aun cuando ésta no esté reconocida en horas de trabajo, remuneración o reconocimiento social.

\section{Sentido de la vocación}

Este reconocimiento trascendental y esta orientación prosocial que caracterizan a la vocación son los que, a su vez, otorgan sentido (o propósito) a las vidas de los académicos y a su práctica profesional, puesto que no sólo ejercen la profesión "para la que han sido llamados" sino que, además, experimentan una elevada satisfacción por ayudar a otros. Como lo evidencian los relatos a continuación, esta percepción de ayuda a otros se presenta en la vida profesional de los académicos a diferentes niveles del vínculo social, que van desde las relaciones sociales más cercanas y concretas, hacia las más distantes e intangibles.

\section{Vínculo social inmediato}

Así, por un lado, se percibe que el sentido de vocación está dado por la ayuda otorgada a personas directamente involucradas en la labor académica del participante (tales como estudiantes) y por ello estas percepciones se relacionan con un nivel local, o afin/ cercano al académico. De esta forma, el docente universitario explica que su trabajo tiene un impacto significativo sobre la vida de las personas con las 
que se relaciona de manera directa en su quehacer académico.

Para mí es un rol de satisfacción, es grato y lo elijo [...] está formando un futuro, tenés un rol chiquito digamos $[\ldots]$ que está formando a una persona que se va a recibir y es una función muy importante (ejemplo 13, entrevista 10).

Placer, realmente me encanta porque siento que estoy haciendo una diferencia, siento que estoy aportando algo [...] Precisamente, porque pueden modificar, pueden aportarle a otra persona algo para mejorar su calidad de vida y al mismo tiempo, si esa persona es un profesional como van a ser los estudiantes, que son futuros profesionales, pueden ellos mejorar la calidad de vida de otras personas también (ejemplo 14, entrevista 11).

La vuelta que tengo de los alumnos, el agradecimiento. Tengo alumnos que han sido residentes míos hace 20 años y que se han ido a Europa, tengo gente en Barcelona, tengo gente en Londres y todos los fines de año me escriben felicitándome por el año que viene y hace más de 20 años que se recibieron $\mathrm{y}$ fueron mis primeros residentes, o sea que eso me da la pauta de que hay una trascendencia en lo que uno hace, no se queda en uno solamente (ejemplo 15, entrevista 11).

El impacto social alcanzado en esta instancia es claramente visible para los académicos, quienes parecen retroalimentar su sentimiento de vocación a través del sentido que adquiere su práctica académica en la formación de futuros profesionales.

\section{Vínculo social periférico}

Por otro lado, se percibe que el sentido de vocación está dado por la ayuda otorgada a personas indirectamente involucradas en la labor académica y por ello estas percepciones se relacionan a un nivel periférico del vínculo social del académico. Este vínculo está dado por el aporte que el docente siente que realiza de forma indirecta en la comunidad local y la sociedad de la cual forma parte, a través del impacto que su rol tiene en la formación universitaria de profesionales que cumplirán roles significativos en la sociedad una vez graduados.

Lo que más me gusta es la formación de graduados, allí hace dos años que estoy dirigiendo ese programa, ese programa tiene una pata en posgrado y una pata en extensión, porque allí formamos a gente que esta graduada para que atienda pacientes que están en [una] situación con sus derechos vulnerados, entonces de alguna manera como es un programa gratuito, formamos graduados y a su vez esos graduados asisten a personas que no tienen acceso al tratamiento psicológico, ésa es la tarea que desarrollo hoy en día que más me gusta $[\ldots]$ ahí estás formando o trabajando con personas graduadas que están atendiendo ya pacientes, como que el nivel de compromiso es aún mayor (ejemplo 16, entrevista 13).

Un cambio trascendente [...] es el objetivo primario de una casa de altos estudios, hacer cambios que impacten en la sociedad, es esa la función de la Universidad [...] Y obviamente que la unidad académica impacte en el profesional que está formando, entonces el profesional va a impactar en la sociedad y así sucesivamente. Tiene que ser un bucle virtuoso (ejemplo 17, entrevista 1).

Yo creo que el docente en general en cualquier nivel educativo tiene un papel central, porque es allí donde más uno puede contribuir a la formación de un ciudadano libre, crítico, y eso repercute en todos los niveles sociales, en todos los niveles de intercambio y de relaciones sociales (ejemplo 18, entrevista 15).

Este sentido de entrega, de aporte al crecimiento profesional de una persona y al desarrollo de la comunidad que los rodea está motivado por una pasión por la disciplina [...] que ayuda a generar una sensación de bienestar general, como un intento de transformar ciertas realidades caracterizadas por la injusticia social, como 
explican las entrevistas 1 y 18. Así, como se menciona también en el segundo punto de este análisis, el sentido de vocación adquiere un valor social altruista al concebir que uno de los propósitos de la profesión docente es causar un impacto positivo en las esferas más distantes de la sociedad.

\section{Vínculo imaginario}

Por último, los académicos participantes explican que el sentido de vocación también está constituido por la contribución significativa que éstos realizan a la generación de nuevo conocimiento.

Es una motivación que tiene que ver con una, a ver $[\ldots]$ con una realización desde lo personal $[\ldots]$ donde [en] la universidad vos tenés la posibilidad justamente de producir conocimientos en sí mismo y ése es el objetivo, a diferencia de una práctica privada donde el objetivo será otro si trabajas, yo trabajo en psicología laboral, puede ser el cliente, puede ser la persona que busca una orientación, lo ayuda la intervención, pero no es el conocimiento a veces en sí mismo. El estar en la universidad tiene esa prioridad que es producir conocimientos (ejemplo 19, entrevista 10).

La creación de nuevo conocimiento está asociada al vínculo que el académico establece con la contribución que realiza a nivel disciplinar y lo vincula a la misión última de la universidad desde el punto de vista del rol universal de la institución. Desde esa perspectiva, el sentido de vocación está relacionado con la pertenencia disciplinar, es decir, con la membrecía a una comunidad de práctica imaginaria (en términos de Norton, 2001 y Wenger, 2002) para la cual el académico crea conocimiento dentro de un área determinada de la ciencia.

A continuación, se presenta un diagrama que resume las (sub-)categorías identificadas en los acápites precedentes. En éste se muestra cómo los diferentes aspectos constitutivos de las tres macro-categorías exploradas de acuerdo con los relatos de estos académicos se vinculan con el constructo de vocación para la carrera académica.

\section{Figura 1. Elementos constitutivos de la vocación académica}

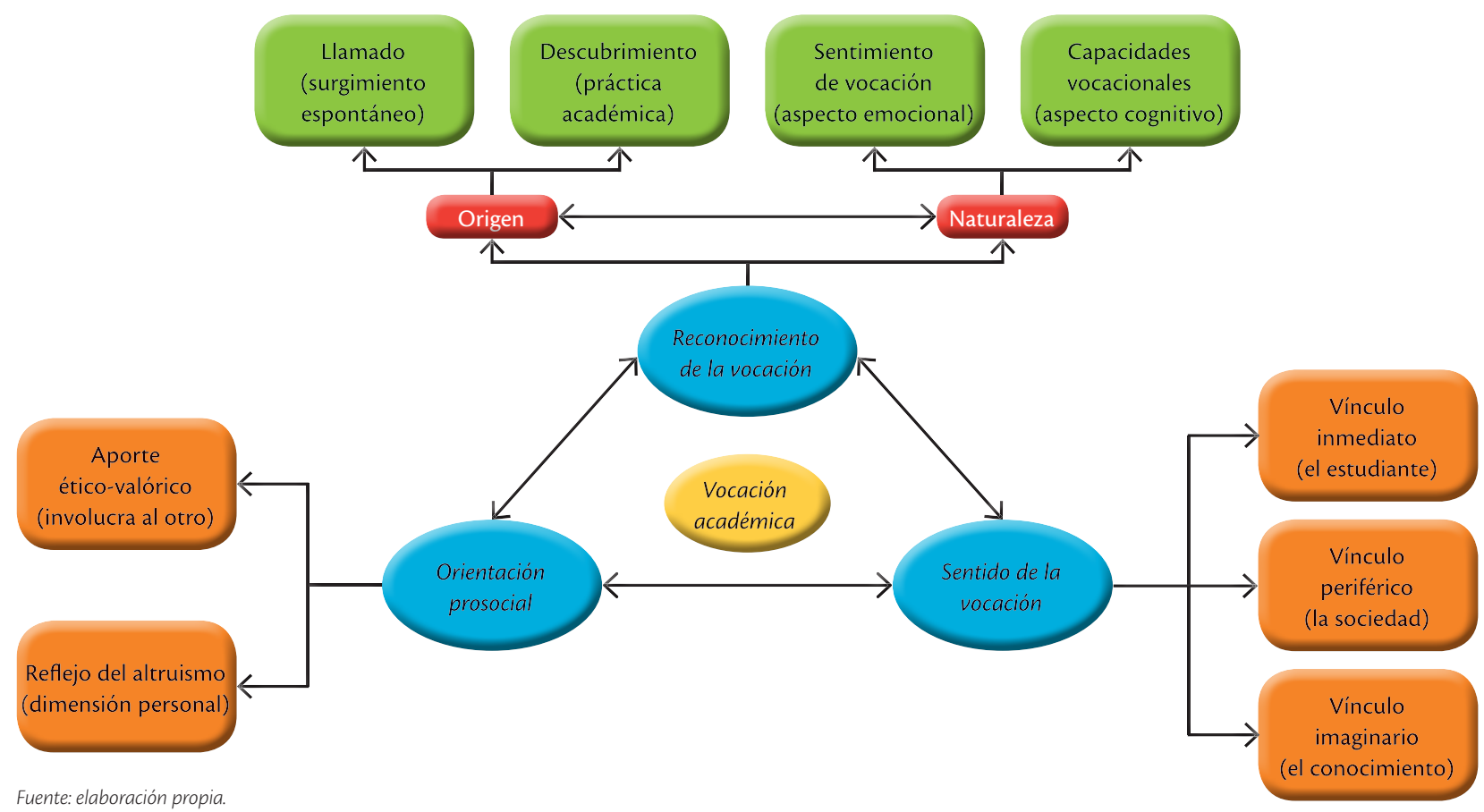




\section{Discusión}

La literatura tradicionalmente ha señalado que la vocación de carrera asume un rol fundamental en la profesión académica (Fuentes, 2001). De hecho, son varias las investigaciones que sugieren que la vocación por el ejercicio de la profesión es uno de los elementos que protegen a los docentes universitarios de los elevados riesgos psicosociales que caracterizan su trabajo, al brindarles la oportunidad de obtener una profunda sensación de sentido, propósito y significado (Pujol-Cols et al., 2019). Sin embargo, a pesar de constituir un tema de gran relevancia, son muy pocas las investigaciones que han examinado la vocación desde el punto de vista empírico, especialmente en el contexto de la profesión docente (Duffy et al., 2011). Más aún, la mayor parte de estos estudios se han efectuado desde un abordaje cuantitativo, con el propósito de describir un conjunto de dimensiones genéricas de la vocación y relacionarlas con algunos estados afectivos o conductuales de los docentes (Duffy et al., 2013; 2018). Es así que este estudio tuvo por propósito proponer una serie de sub-dimensiones que ayudan a operacionalizar las macro-categorías del concepto de vocación para la carrera académica, de modo de reflejar la complejidad del constructo en este contexto profesional. A tal fin, se adoptó un abordaje cualitativo consistente en el análisis de los relatos de un grupo de académicos de una universidad pública de Argentina.

De los análisis realizados emergió que algunos de los docentes universitarios reconocen su vocación de carrera a través de procesos involuntarios, no intencionados y espontáneos (lo que aquí denominamos llamado vocacional), mientras que otros académicos la descubren al atravesar una fase de exploración y búsqueda intencionada por la que entran en contacto formal con la carrera docente (lo que aquí llamamos el descubrimiento vocacional). Estos hallazgos resultan consistentes con las proposiciones de Duffy y Dik (2009) que señalan que la vocación de carrera puede emerger tanto de una fuente interna (es decir, aquella que se ve originada dentro del individuo, donde la satisfacción individual representa la motivación principal) como de una fuente externa (es decir, aquella que tiene su origen en alguien o en un evento externo al individuo). Independientemente del proceso que dé origen al reconocimiento, los académicos, a su vez, desarrollan lazos de vinculación emocional con su profesión (aspecto emocional de la vocación), a la vez que descubren sus propias capacidades y competencias para el ejercicio profesional (aspecto cognitivo de la vocación).

Otro de los elementos que componen la vocación para la carrera académica es la orientación prosocial (Mesurado et al., 2019). Desde la perspectiva del otro, la vocación implica un aporte ético-valorativo que se manifiesta en la oportunidad de formar profesionales en valores, pensamientos y acciones éticas con las que los académicos se sienten personalmente identificados y que perciben como socialmente deseables y necesarias. Asimismo, de esta investigación surge que la orientación prosocial también apoya el desarrollo personal de los académicos, el cual pareciera alejarse de un interés en las recompensas económicas (reflejo del altruismo). Quizás por este motivo, varias investigaciones previas reportaron que la remuneración y otras compensaciones materiales asumen un rol poco relevante a la hora de explicar el bienestar de los docentes argentinos (Pujol-Cols et al., 2019), a pesar de constituir una de las profesiones peor remuneradas a nivel mundial (Groisman y García de Fanelli, 2009; ver también Centro de Estudios de la Educación Argentina, 2018).

Finalmente, el reconocimiento y la orientación prosocial que caracterizan a la vocación son los que, a su vez, otorgan sentido y propósito a las vidas de los académicos y a su práctica profesional. La docencia, específicamente, ha sido definida como una profesión de elevado contenido vocacional, pues, como señala Fuentes (2001), los profesionales docentes suelen sentirse llamados a asumir este rol con una responsabilidad y pasión que trasciende sus 
propias necesidades e intereses individuales. Como lo evidencian los hallazgos de esta investigación, el ejercicio de este rol se encuentra fuertemente orientado hacia una comunidad con la que los docentes se sienten personalmente comprometidos (considerar Duffy et al., 2018). Esta percepción de ayuda a otros se presenta en la vida profesional de los académicos en diferentes niveles del vínculo social que van desde las relaciones sociales más cercanas y concretas, hacia las más distantes e intangibles.

Si bien creemos que esta investigación realiza un aporte importante al conocimiento sobre los elementos que componen la vocación de carrera, particularmente en el contexto de la profesión académica en Argentina, es necesario reconocer sus limitaciones, de modo que sea posible propiciar el desarrollo de futuras investigaciones en la temática. En primer lugar, este estudio fue mayormente exploratorio e incluyó académicos de una misma

\section{Referencias}

Avey, James B., Rebecca J. Reichard, Fred Luthans y Ketan H. Mhatre (2011), "Meta-analysis of the impact of positive psychological capital on employee attitudes, behaviors, and performance", Human resource Development Quarterly, vol. 2, núm. 22, pp. 127-152.

Cardador, M. Teresa, Erik Dane y Michael G. Pratt (2011), "Linking calling orientations to organizational attachment via organizational instrumentality", fournal of Vocational Behavior, vol. 79, núm. 2, pp. 367-378.

Centro de Estudios de la Educación Argentina (2018), Los salarios docentes en el mundo y en Argentina, Buenos Aires, Universidad de Belgrano.

Collado, Patricia Alejandra, Cecilia Beatriz Soria, Eliana CanafogliaySandraAliciaCollado(2016), "Condiciones de trabajo y salud en docentes universitarios y de enseñanza media de Mendoza, Argentina: entre el compromiso y el desgaste emocional", Salud Colectiva, núm. 12, pp. 203-220. universidad argentina. Si bien la presente investigación no pretende realizar generalizaciones atribuibles al conjunto del sistema universitario argentino, futuros estudios que incluyan participantes de otras universidades serían fundamentales para validar los hallazgos obtenidos. Por último, este estudio se focalizó exclusivamente en los elementos que componen la vocación de carrera en el contexto de la profesión académica en Argentina. Futuras investigaciones deberían examinar los mecanismos y procesos por los que la vocación interactúa con los diferentes recursos y demandas laborales que caracterizan la profesión docente, así como los efectos de estas dinámicas sobre el bienestar psicosocial.

\section{Agradecimientos}

Los autores agradecen a la Lic. Cynthia Navarro por su gran colaboración en el proceso de recolección de datos.

Douglass, Richard P., Ryan D. Duffy y Kelsey L. Autin (2016), "Living a calling, nationality, and life satisfaction: a moderated, multiple mediator model", Journal of Career Assessment, vol. 24, núm. 2, pp. 253-269.

Dik, Bryan J., Brandy M. Eldridge, Michael F. Steger y Ryan D. Duffy (2012), "Development and validation of the calling and vocation questionnaire (CVQ) and brief calling scale (BCS)", Fournal of Career Assessment, vol. 20, núm. 3, pp. 242-263.

Dobrow, Shoshana R. y Jennifer Tosti-Kharas (2012), "Listen to your heart? Calling and receptivity to career advice", Fournal of Career Assessment, vol. 20, núm. 3, pp. 264-280.

Duffy, Ryan D. y Bryan J. Dik (2013), "Research on calling: What have we learned and where are we going?", fournal of Vocational Behavior, vol. 83, núm. 3, pp. 428-436.

Duffy, Ryan D. y Bryan J. Dik (2009), "Beyond the self: External influences in the career development process", The Career Development Quarterly, vol. 58, núm. 1, pp. 2943. 
Duffy, Ryan D., Bryan J. Dik y Michael F. Steger (2011), "Calling and work-related outcomes: career commitment as a mediator", fournal of Vocational Behavior, vol. 78, núm. 2, pp. 210-218.

Duffy, Ryan D., Elizabeth M. Bott, Blake A. Allan, Carrie L. Torrey y Bryan J. Dik (2012), "Perceiving a calling, living a calling, and job satisfaction: testing a moderated, multiple mediator model", fournal of Counseling Psychology, vol. 59, núm. 1, pp. 50-59.

Duffy, Ryan D., Kelsey L. Autin, Blake A. Allan y Richard P. Douglass (2015), "Assessing work as a calling: An evaluation of instruments and practice recommendations", Journal of Career Assessment, vol. 23, núm. 3, pp. 351-366.

Duffy, Ryan D., Bryan J. Dik, Richard P. Douglass, Jessica W. England y Brandon L. Velez (2018), "Work as a calling: A theoretical model", fournal of Counseling Psychology, vol. 65, núm. 4, pp. 423-439.

Eisenhardt, K. M. (1989), "Building theories from case study research", Academy of Management Review, 14(4), 532-550.

Extremera, Natalio, Auxiliadora Durán y Lourdes Rey (2010a), "Recursos personales, síndrome de estar quemado por el trabajo y sintomatología asociada al estrés en docentes de enseñanza primaria y secundaria”, Ansiedad y Estrés, vol. 16, núm. 1, pp. 47-60.

Extremera, N., L. Rey y M. Pena (2010b), "La docencia perjudica seriamente la salud. Análisis de los síntomas asociados al estrés docente", Boletín de Psicología, vol. 100, núm. 1, pp. 43-54.

Fuentes, Teresa (2001), "La vocación docente: una experiencia vital", Ars Brevis, vol. 7, núm. 1, pp. 285-303.

García de Fanelli, A. G. (2016), "Informe nacional: Argentina", en J. J. Brunner (ed.), Educación Superior en Iberoamérica-Informe 2016, Santiago de Chile, Centro Universitario de Desarrollo (CINDA).

Goh, Zen, Remus Ilies y Kelly Schwind Wilson (2015), "Supportive supervisors improve employees' daily lives: The role supervisors play in the impact of daily workload on life satisfaction via work-family conflict", Journal of Vocational Behavior, vol. 89, núm. 1, pp. 65-73.
Groisman, Fernando y Ana M. García de Fanelli (2009), "Incentivos a la profesión académica: los salarios de los docentes universitarios en la Argentina", Revista Latinoamericana de Estudios del Trabajo, vol. 14, núm. 21, pp. 143-167.

Hentrich, Stephan, Andreas Zimber, Nadia SosnowskyWaschek, Sabine Gregersen y Franz Petermann (2017), "The role of core self-evaluations in explaining depression and work engagement among managers", Current Psychology, vol. 36, núm. 3, pp. 516-529.

Kammeyer-Mueller, John D., Timothy A. Judge y Brent A. Scott. (2009), "The role of core self-evaluations in the coping process", fournal of Applied Psychology, vol. 94, pp. 177-195.

Lazzaro-Salazar, Mariana (2019), "Researchers as brokers: ethical reflections from a study of migrant physicians in Chile", The Social Science Journal, vol. 56, pp. 609-616.

Lazzaro-Salazar, Mariana (2013), "Investigating professional identity construction in intercultural health settings in New Zealand", Ph.D. dissertation, Victoria University of Wellington.

Littman-Ovadia, Hadassah y Michael Steger (2010), "Character strengths and well-being among volunteers and employees: Toward an integrative model", The Fournal of Positive Psychology, vol. 5, núm. 6, pp. 419-430.

Luthans, Fred y Carolyn M. Youssef (2004), "Human, social, and now positive psychological capital management: investing in people for competitive advantage", Organizational Dynamics, vol. 33, núm. 2, pp. 143-160.

Marquina, Mónica (2012), "La profesión académica en Argentina: principales características a partir de las políticas recientes", en El futuro de la profesión académica: desafios para los países emergentes, Sáenz Peña, EDUNTREF, pp. 126-147.

Meliá, José Luis, Clotilde Nogareda, Matilde Lahera, Antonio Duro, José María Peiró, Marisa Salanova y Diego Gracia (2006), "Principios comunes para la evaluación de los riesgos psicosociales en la empresa", en Perspectivas de intervención en riesgos psicosociales. Evaluación de riesgos, Barcelona, Foment del Treball Nacional, pp. 13-36. 
Mendoza, Ilsa y Mariana Lazzaro-Salazar (2018), "Análisis de la construcción de identidad del inmigrante en los Programas de gobierno de la presidente Michelle Bachelet", Papeles de Trabajo, vol. 36, núm. 1, pp. 65-80. Mesurado, Belén, María José Distéfano y Mariana Falco (2019), "Estudio de usabilidad y percepción de eficacia de los módulos que componen el Programa Héroe", Estudios sobre Educación, núm. 37, pp. 11-32.

Newman, Alexander, Deniz Ucbasaran, F. E. I. Zhu y Giles Hirst. (2014), "Psychological capital: a review and synthesis", Fournal of Organizational Behavior, vol. 35, núm. 1, pp. 120-138.

Norton, Bonny (2001), "Non-participation, imagined communities and the language classroom", Learner contributions to language learning: New directions in research, vol. 6, núm. 2, pp. 159-171.

Pujol-Cols, Lucas y Mariana Lazzaro-Salazar (2018), "Psychosocial risks and job satisfaction in Argentinian scholars: exploring the moderating role of work engagement", Journal of Work and Organizational Psychology, vol. 34, núm. 3, pp. 145-156.

Pujol-Cols, Lucas y Mariana Arraigada (2017), "Propiedades psicométricas del Cuestionario de Riesgos Psicosociales Copsoq-Istas 21 y aplicación en docentes universitarios argentinos", Cuadernos de Administración, vol. 30, núm. 55, pp. 97-125.

Pujol-Cols, Lucas, Mariana Foutel y Luis Porta (2019), "Riesgos psicosociales en la profesión académica: un análisis interpretativo del discurso de docentes universitarios argentinos", Trabajo y Sociedad, vol. 20, núm. 33, pp. 197-223.

Rumbley, Laura, Iván Francisco Pacheco y Philip G. Altbach (2005), “¿Cómo se relacionan los obstáculos y facilitadores organizacionales con el burnout docente?: un estudio longitudinal", Revista de Psicología del Trabajo y de las Organizaciones, vol. 21, núm. 1-2, pp. 37-54.

Salanova, M., I. M. Martínez y L. Lorente (2005), “CCómo se relacionan los obstáculos y facilitadores organizacionales con el burnout docente?: un estudio longitudinal”, Revista de Psicología del Trabajo y de las Organizaciones, 21(1-2), 37-54.

Shin, Jung Cheol y Jisun Jung (2014), "Academics job satisfaction and job stress across countries in the changing academic environments", Higher Education, vol. 67, pp. 603-620.

Times Higher Education (2010), "Worldwide Academic Job Satisfaction", https://www.insidehighered.com/ news / 2010/07/02/worldwide-academic-jobsatisfaction .

Weir, Kirsten (2013), "More than job satisfaction", APA Monitor on Psychology, vol. 44, pp. 39.

Wegner, Phillip (2002), Imaginary communities: utopia, the nation, and the spatial histories of modernity, California, University of California Press.

Wrzesniewski, Amy, Clark McCauley, Paul Rozin y Barry Schwartz (1997), "Jobs, careers, and callings: people's relations to their work", fournal of Research in Personality, vol. 31, núm. 1, pp. 21-33.

Xanthopoulou, Despoina, Arnold B. Bakker, Evangelia Demerouti y Wilmar B. Schaufeli (2009), "Reciprocal relationships between job resources, personal resources, and work engagement", Journal of Vocational Behavior, vol. 74, núm. 3, pp. 235-244.

Yin, Hong-biao, John Chi Kin Lee y Zhong-hua Zhang (2013), "Exploring the relationship among teachers' emotional intelligence, emotional labor strategies and teaching satisfaction", Teaching and Teacher Education, vol. 35, pp. 137-145.

\section{Cómo citar este artículo:}

Pujol-Cols, Lucas y Mariana Lazzaro-Salazar (2021), "Vocación de carrera en académicos de una universidad pública en Argentina”, Revista Iberoamericana de Educación Superior (RIES), vol. XII, núm. 34, pp. 72-86, DoI: https://doi.org/10.22201/iisue.20072872e.2021.34.979 [Consulta: fecha de última consulta]. 